J.A. JENKINS

KODAI MATH. J.

15 (1992), $79-81$

\title{
A CRITERION ASSOCIATED WITH THE SCHLICHT BLOCH CONSTANT
}

\author{
By James A. JENKins
}

1. Let $f \in S$ map the unit disc onto the domain $D_{f}$ and let $B_{f}$ be the least upper bound of the radii of discs lying in $D_{f}$. It follows at once from the $\frac{1}{4}$-theorem that $B_{f}$ has a positive lower bound for all $f \in S$. Let $\alpha=$ g.l.b. $B_{f}$. By a compactness argument it follows that there exist functions for which this is attained. $\alpha$ is called the schlicht Bloch constant. We call a function $f$ for which $B_{f}=\alpha$ an extremal function for this problem and the corresponding $D_{f}$ an extremal domain. A disc of radius $\alpha$ in $D_{f}$ is then called an extremal disc.

Some years ago I discovered a criterion for an extremal domain which eliminates many examples used in estimating $\alpha$ from above from providing extremal functions, for example that given by Ruth Goodman. Not long ago I mentioned this criterion to Hummel and a somewhat vague version of it is quoted in the paper [1]. Since that presentation is not really adequate it seems desirable to give a complete account.

The criterion is an easy consequence of a result of Lavrentiev [4] which also is quoted in the article of Golusin [2]. We formulate this result in the following theorem.

THEOREM. Let $R$ denote the circular $r i n g ~ 1<|w|<r$ and let $p$ be a point satisfying $1<p<r$. Then the maximal reduced module with respect to $p$ of $a$ simply-connected domain containing $p$ and contained in $R$ is attained uniquely for the domain obtained by slitting $R$ along the segment $-r<w<-1$.

We indicate the very simple proof given in $[2,4] . R$ can be mapped conformally on the doubly-connected domain bounded by the segment $\left[\frac{1}{4}, a\right]$ and the ray on the positive real axis $[b, \infty], \frac{1}{4}<a<b$, with $p$ going to the origin. The result then follows at once from the $\frac{1}{4}$-theorem, see for example [3, Theorem 6.1].

This research is supported in part by the National Science Foundation.

Received May 13, 1991. 
This result can also be proved very easily by the method of the extremal metric.

2. We now state the desired criterion.

CRITERION. Let $D_{f}$ be an extremal domain whose boundary contains a rectilinear segment $\sigma$ on which there is a non-endpoint $P$ with $s>0$ such that

$$
\{|z-P|<s\}-\sigma \subset D_{f}
$$

and such that $P$ is not on the circumference of an extremal disc. Then $\sigma$ must lie on a ray emanating from the origin and $D_{f}$ is symmetric under reflection in this ray.

Indeed removing a sufficiently small open subsegment $\sigma^{\prime}$ of $\sigma$ containing $P$ we obtain a doubly-connected domain $\mathscr{D}$ which contains no disc of radius greater than $\alpha$. Mapping $\mathscr{D}$ on a ring $1<|w|<r$ so that the origin goes to a point $p$ with $1<p<r$ we see that the image of $\sigma^{\prime}$ must be the open segment $-r<w<-1$ since otherwise replacing $\sigma^{\prime}$ by the inverse image of that open segment we would obtain a simply-connected domain containing no disc of radius greater than $\alpha$ which would be the image of the unit disc under a conformal mapping preserving the origin and with derivative of modulus greater that one at that point. This contradicts the extremal nature of $D_{f}$.

By a completely analogous proof we obtain the following criterion.

CRITERION'. Let $D_{f}$ be an extremal domain whose boundary contains an arc $\tau$ on a circumference $\gamma$ on which there is a non-endpoint $P$ with $t>0$ such that

$$
\{|z-P|<t\}-\tau \subset D_{f} .
$$

and such that $P$ is not on the circumference of an extremal disc. Then the origin must lie on $\gamma$ and $D_{f}$ is symmetric under reflection in $\gamma$.

\section{BIBLIOGRAPHY}

[1] E. Beller and J. Hummel, On the univalent Bloch constant, Complex Variables, vol. 4 , 1985, pp. 243-252.

[2] G. M. Golusin, Interior problems of the theory of univalent functions, Uspekhi Mathematicheskii Nauk, vol. 6, 1939, pp. 26-89, (Russian) (Translated under auspices of Office of Naval Research 1947.)

[3] James A. Jenkins, Univalent Fuunctions and Conformal Mapping, Springer Verlag, Berlin-Göttingen-Heidelberg, 1958.

[4] M. A. Lavrentiev, On the theory of conformal mappings, Trudy Matematicheskogo Instituta imeni V. A. Steklova, No. 5, 1934, pp. 159-245, (Russian) (Translated in A.M. S. Translations, vol. 122, 1984, pp. 1-63.) 
WASHINGTON UNIVERSITY

Campus Box 1146

One Brookings Drive

St. Louis, Missouri 63130-4899

U.S.A. 